\title{
FEMINISMO, GÉNERO Y LABORES REPRODUCTIVAS
}

\section{Carmen Benedito Pérez}

Universitat Politècnica de València. Programa de Doctorado en Arte: Producción e Investigación

\section{Resumen}

El artículo consta de tres partes, la primera una introducción en la que se reflexiona sobre la necesidad de estudiar la diferencia que existe entre sexos respecto de las labores reproductivas, y la importancia de comprender las nuevas narrativas y necesidades que se van generando respecto al tema de la reproducción y maternidad, una segunda parte en la que se exponen cuatro artistas que nos han aportado narrativa plástica, visual y vivencial sobre la maternidad y en la que se valoran los cambios estéticos en la interpretación del tema y una última parte en la que extraen conclusiones y se formulan hipótesis de posibles alternativas para la integración de la reproducción humana y social en un nuevo organismo social.

\section{Palabras-clave: ARTE-VIDA; MATERNIDAD; GÉNERO; PRODUCCIÓN-RE- PRODUCCIÓN; REPRODUCCIÓN SOCIAL}

\section{FEMINISM, GENDER AND REPRODUCTIVE WORK}

\section{Abstract}

The article consists of three parts, the first an introduction which speaks about of the necessity of studying the difference that exists between the sexes about reproductive tasks and the importance of understanding the new narratives and necessities that are generated on the issue of the reproduction and motherhood, a second part in which four artists are exposed who have contributed plastic, visual and experiential narrative on motherhood and in which aesthetic changes in the interpretation of the theme are valued and a final part in which conclusions are drawn and hypotheses are formulated for possible alternatives to the integration of human and social reproduction into a new social organism.

\section{Keywords: ART-LIFE; MOTHEHOOD; GENDER; PRODUCTION-REPRO- DUCTION; SOCIAL REPRODUCTION}

\footnotetext{
Benedito Pérez, Carmen. 2017 . "Feminismo, género y labores reproductivas". AusArt 5 (1): 103-115 D0I: 10.1387/ausart.17782
}

\section{AUSART}




\section{UNA PERFUSIÓN NECESARIA}

"Sabemos mucho más acerca del aire que respiramos o de los mares que atravesamos que acerca de la naturaleza y del significado de maternidad"1

Adrienne Rich

Desde los años 60 y 70 el feminismo y la teoría de género junto con los avances tecnológicos en temas de salud sexual y anticoncepción entre otros factores, están originando una reorganización social en la que está cambiando el modelo de familia.

La incorporación de la mujer al mercado laboral, la política, la comunidad científica, la academia y las artes entre otros ámbitos, están creando una nueva forma de comprender la vida, hoy en día las mujeres en Occidente, hablando de forma general, tienen más libertad en cuanto a la elección de pareja y la de ser o no madres.

La diferencia fundamental entre los sexos ha sido y sigue siendo la de las labores reproductivas, la gestación, el parto y la lactancia materna sólo pueden acometerlo las mujeres XX (¿hembras?). Las labores de cuidados en la reproducción social en este momento siguen recayendo en su mayor parte en las madres (Legarretalza 2009), que derivan funciones por falta de tiempo a su red familiar $\mathrm{y} / \mathrm{o}$ a instituciones públicas o privadas, importante es mencionar que en el sistema económico actual no se contemplan como productivas las labores de reproducción y cuidados, disfunción del sistema que provoca una situación de discriminación económica estructural².

Maternidad y paternidad son conceptos que se encuentran en un vórtice en este momento, con infinidad de elementos girando a gran velocidad, investigación con células madre, embrionarias, clonación, reproducción asistida, aborto, úteros artificiales, vientres de alquiler, regulaciones legales, custodia de los hijos, pruebas de ADN.

El gran reto en el presente es el de analizar las labores de reproducción con el fin de encontrar soluciones que permitan un desarrollo individual satisfactorio y que, a la vez, favorezcan un desarrollo social y de conjunto respetuoso, justo, armónico y sostenible para niños y adultos. Para ello, es necesario investigar para obtener conocimiento sobre hasta dónde llega la construcción social y cultural del género y hasta dónde llegan las necesidades matéricas inherentes 
de cada sexo, XX XY, así como las necesidades matéricas de la infancia, y como estas necesidades matéricas y estas construcciones se retroalimentan.

\section{ARTE Y MATERNIDAD, MATERNIDAD Y ARTE}

Siguiendo una línea de tiempo en el Arte moderno y contemporáneo hasta el día de hoy a continuación se detallo una serie de artistas que han aportado o aportan actualmente narrativa escrita, plástica, visual y vivencial al tema y que han manifestado públicamente su opinión o han interpretado esta realidad desde los años sesenta y setenta hasta el día de hoy.

He de mencionar que cada vez más, hay mujeres que se dedican al mundo del arte y que trabajan con interpretaciones de la maternidad, he seleccionado cuatro de las que hablo a continuación dado que son las que más se mencionan en redes en relación con el tema objeto de mi investigación en curso.

\section{LOUISE BOURGEOIS, 1911-2010}

Artista francesa, en las vanguardias interpretó su realidad biográfica mediante la pintura, grabado, escultura e instalaciones. Temas recurrentes en su producción artística fueron género y sexualidad.

En la última década de su vida abordó la función reproductiva de la mujer, entre sus obras destaco aquí una obra perteneciente a una serie de pinturas en las que utilizaba sobre todo gouache rojo. Louise Bourgeois hablaría de su madre, su padre y sus hijos como sus tres marcos de referencia ${ }^{3}$.

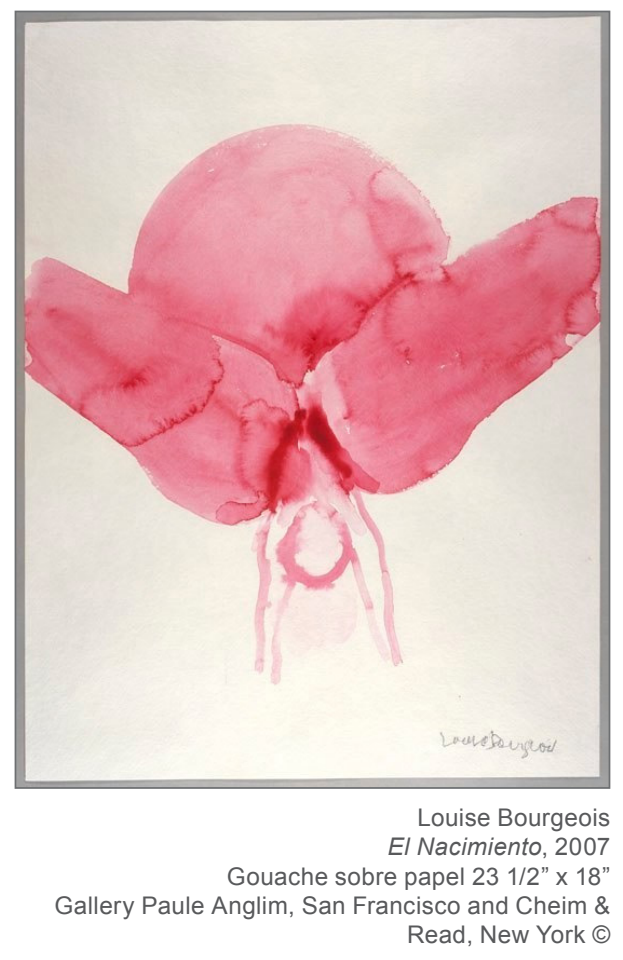




\section{MARY KELLY, 1941}

En Antepartum, 1973 Mary Kelly, artista estadounidense, nos muestra en un vídeo su vientre con los movimientos de su hijo aún no nacido para más tarde en Post-Partum document, en el que durante seis años, explora la relación madre e hijo y nos la presenta con una obra conceptual influenciada por el Feminismo y el psicoanálisis. La artista exploró la contradicción que se le generaba entre el desempeño de su labor artística y su labor como madre, entre sus labores creativas y sus labores reproductivas, estableciendo en su obra una diferencia entre su experiencia y el análisis de la misma.

La obra consta de seis series y contiene más de un centenar de documentos, como ropa usada de su hijo, notas de diario, gráficos y otros objetos tales como pañales usados.

Mary Kelly grabó una serie de conversaciones con su hijo, reflexionó sobre ellas y luego le permitió garabatear una serie de documentos, con ello realizó una serie compuesta de trece obras de lápiz, ceras, tiza y diagramas impresos sobre papel.

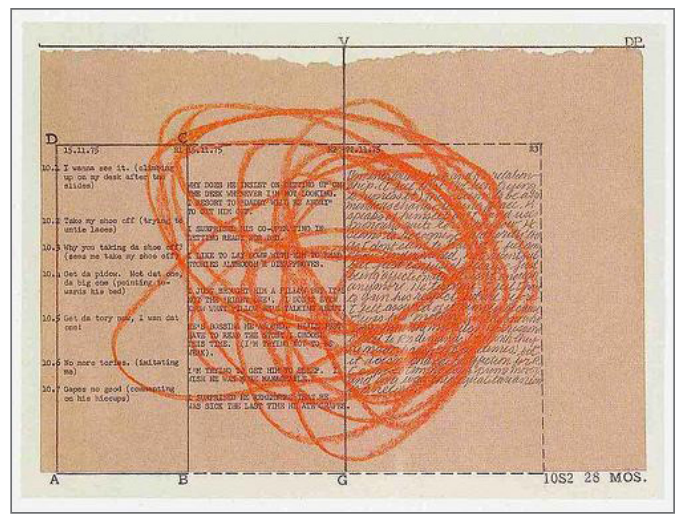

Detalle

cartulina blanca, papel, ceras

1 of the 13 piezas, $35.5 \times 28 \mathrm{~cm}$ cada una Post-Partum Document: Documentation III

Analysed Markings and Diary-perspective Schema, 1975

Tate Modern, London @

\section{RINEKE DIJKSTRA, 1959}

Fotógrafa retratista holandesa quien, entre muchos otros trabajos de centrados en la idendidad, retrató a tres mujeres instantes después de dar a luz a sus criaturas en casa, Julie, Den Haag, Netherlands, February 29 1994; Tecla, Amsterdam, Netherlands May 16 1994; y Saskia, Harderwijk, Netherlands, 
March 16 1994. En su trabajo buscó capturar los sentimientos contradictorios que se producen en una mujer tras el parto.

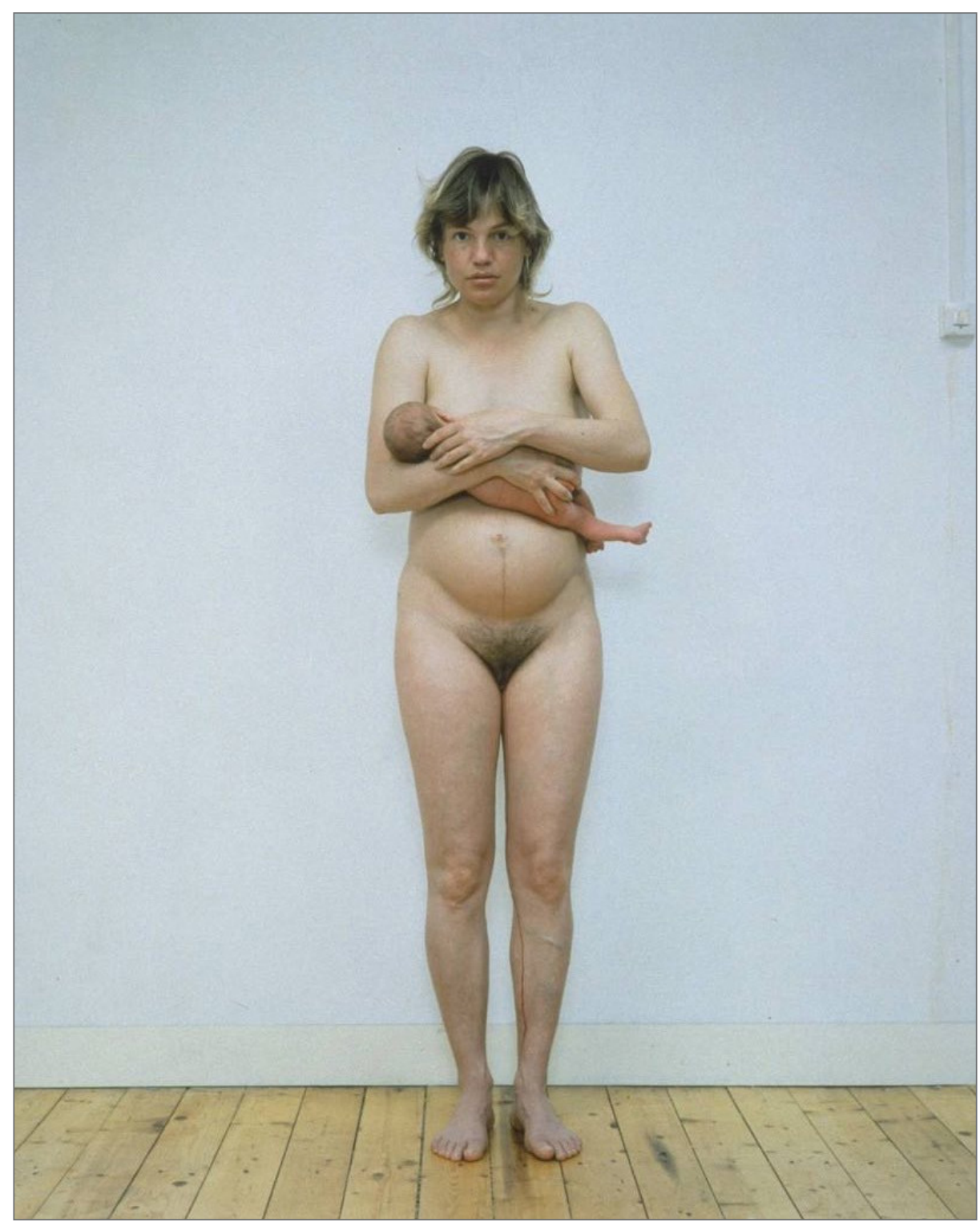

Tecla, Amsterdam, Netherlands, May 16 1994, by Rineke Dijkstra. Marian Goodman Gallery, New York (c) 
Hasta entonces era difícil encontrar representaciones artísticas sobre el parto y su realidad sangrienta, Bill Viola había realizado ya en 1992 una obra consistente en un video con tres proyecciones en una de las cuales aparecía un parto natural filmado en una clínica de California.

\section{ANA ÁLVAREZ-ERRECALDE, 1973}

\section{“El Nacimiento de mi Hija” (2005)}

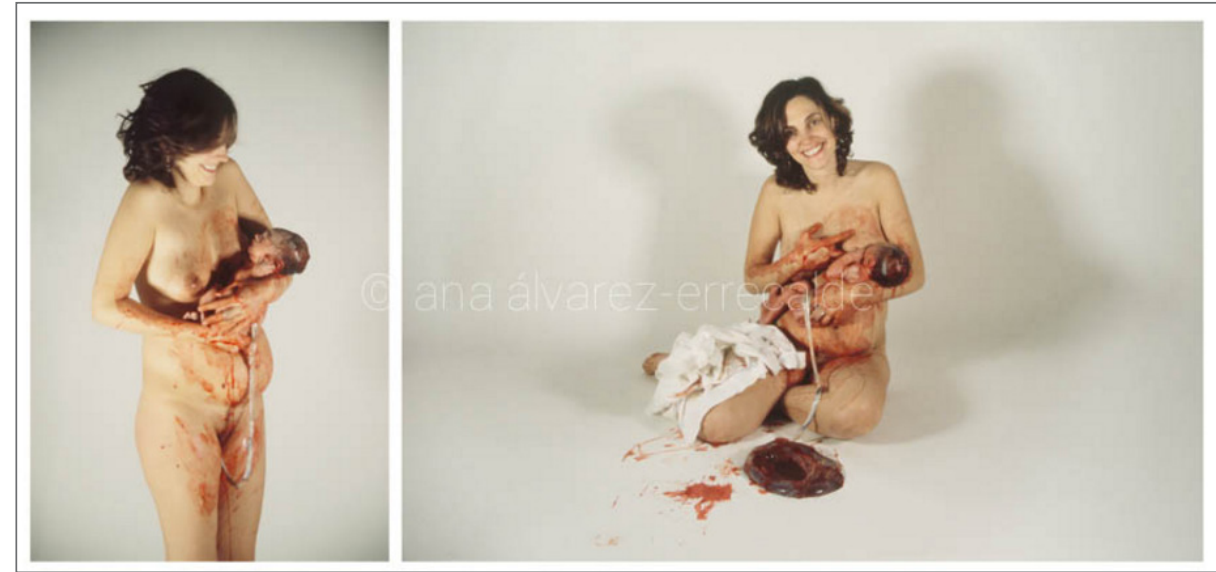

Ana Álvarez-Errecalde (C)

"Con este autorretrato documental (sin photoshop ni técnicas de manipulación de la imagen) en parto quiero desafiar las maternidades "de película" que el cine, la publicidad y la historia del arte enseñan reforzando el estereotipo surgido de las fantasías heterosexuales masculinas que responde a la dualidad madre/puta.

En mi experiencia para parir me abro, me transformo, no soy objeto y sangro, grito y sonrío. Estoy de pie con la placenta aún dentro mío, unida a mi bebé por el cordón umbilical, decido cuándo hacer la foto y mostrarme. Soy protagonista. Soy héroe. Al parir quito el "velo" cultural. Mi maternidad no es virginal ni asép- 
tica. Soy el arquetipo de la mujer-primal, la mujer-bestia que no tiene nada prohibido.

Me alejo de Eva (y el castigo divino de "parirás con el dolor de tu cuerpo") para ver a través de los ojos de Lucy (uno de los primeros homínidos encontrados hasta la fecha)."

(Álvarez-Errecalde 2005).

La artista argentina manifiesta haber descubierto un poder catártico en el arte, trabajando dese su historia personal utilizando la fotografía el vídeo y la instalación.

Muy activa en redes nos habla sobre su trabajo y su vida, es accesible, directa y sincera, al igual que en su trabajo artístico, aporta la interpretación que ella hace de su realidad y está pendiente de lo que sus seguidores le comentan, Álvarez-Errecalde yuxtapone su entorno vital y su capacidad artística e interactúa en la red, lo que hace que se genere conocimiento inmediato desde un enfoque artístico sobre el tema.

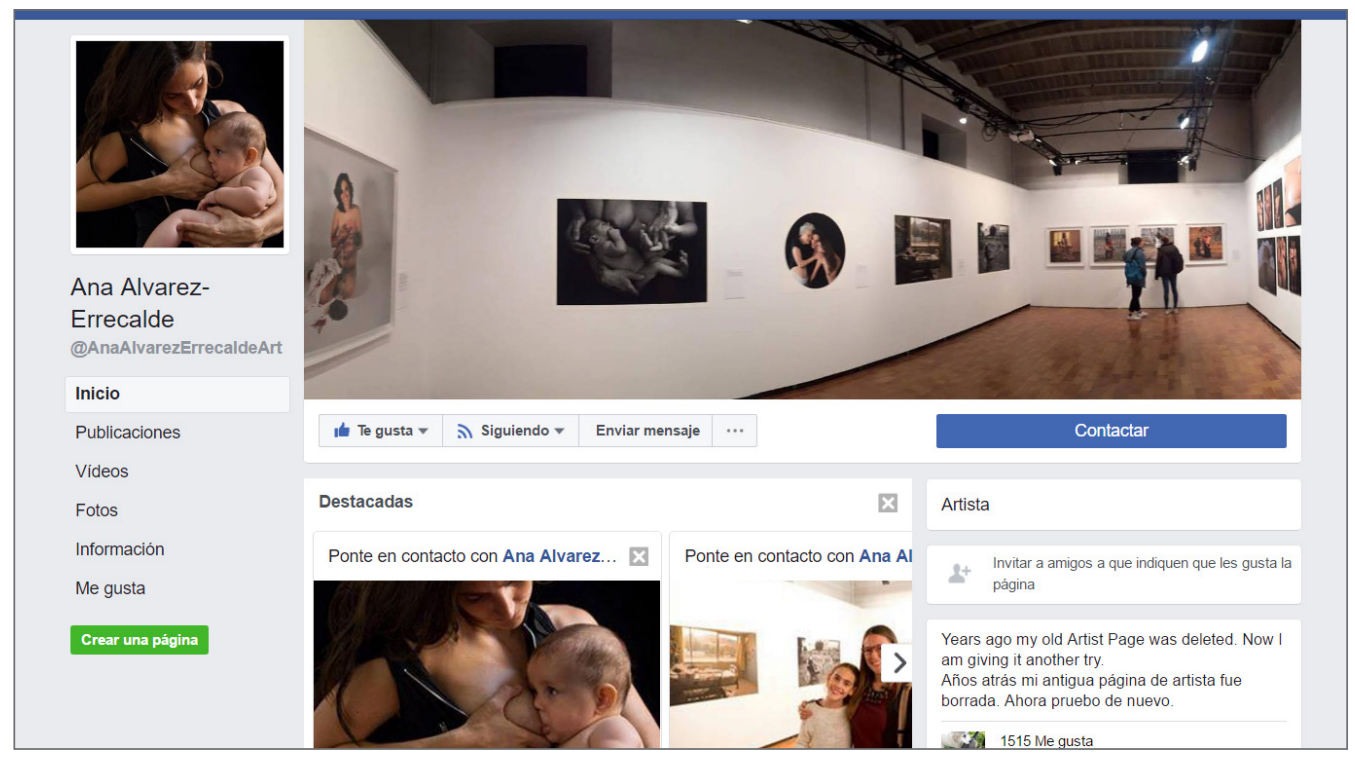

Imagen de la página de Facebook https://www.facebook.com/AnaAlvarezErrecaldeArt/\#

Con esta selección de artistas he pretendido dar una pincelada rápida que dirija un recorrido en el que vaya reflejándose la producción artística del tema de la maternidad, desde los años sesenta y setenta hasta hoy en día. 
Primero Bourgeoise con el gouache rojo sobre el papel, pasamos a Mary Kelly y su obra analítica y conceptual, seguimos con Rineke Dijkstra, que fotografía a madres desnudas con sus bebés en brazos, tras el parto con sangre entre las piernas, captando sus rostros sinceros, desnudando la vulnerabilidad del momento, finalmente encontrarnos con Ana Álvarez-Errecalde, quien se retrata con su hija en brazos justo después del parto y que, con su trabajo y presencia activa en redes apunta el camino en el que se yuxtaponen arte y vida en un mundo de comunicación global.

Artistas como Marina Abramović y Tracey Emin se han manifestado públicamente sobre el tema de la maternidad y otras como Barbara Hepworth, Diane Arbus, Susan Sontag, Margaret Harrison, Susan Hiller, Annie Leibovitz, Maris Bustamante, y Mónica Mayer en Polvo de Gallina negra, Sally Mann, Aleydis Rispa, Elinor Carucci, Ana Casas Broda, María Llopis Navarro, Ana Sabiá y Bea Sánchez entre otras trabajan actualmente o han trabajado en él.

Este fugaz recorrido nos permite percibir la velocidad a la que están aconteciendo los cambios estéticos narrativos sobre un tema que hasta hace poco tiempo había sido tabú y cómo se expande el conocimiento de los temas a través de redes y lo rápido que se retroalimenta la información.

\section{ARTE, MATERNIDAD, COMUNICACIÓN Y VIDA}

Como conclusión podemos afirmar que el tema de la maternidad es asunto cuyas narrativas se expanden a gran velocidad con el uso de las tecnologías sociales ${ }^{4}$ y sus recursos para la comunicación visual y plástica han cambiado desde los años 60 hasta el presente ${ }^{5}$.

Los cuestionamientos que genera el tema eclosionan por necesidad. Parece imprescindible, como nuclear que es, que se analice y se trabaje para engranarlo al sistema con el fin de conseguir el desarrollo de un nuevo organismo social ${ }^{6}$.

Podemos seguir en este modelo social en el que el conocimiento respecto de la reproducción humana y social se autorregula a base de tropiezos ${ }^{7}$ o podemos pensar y teorizar en un modelo en el que se aporte conocimientos básicos al respecto, empezando por cuestiones ligadas a los tiempos de 
dedicación para el proyecto vital de la maternidad-paternidad, desde edades relativamente tempranas.

Lo que parece imprescindible es que los temas de reproducción humana y social sean investigados e impartidos a nivel académico en los ámbitos de las Ciencias Sociales, Psicología, Filosofía y Arte para complementar de forma transversal y mejorar los conocimientos técnicos a nivel reproductivo que se están generando desde el ámbito de la biotecnología ${ }^{8}$.

Lo que la infancia vive determina el futuro, y el cuidado de la infancia necesita mucho tiempo de dedicación, "tiempo que tiene un alto componente relacional" (Legarretalza 2009) por tanto, necesario es pensar en un modelo de futuro en el que la reproducción humana se lleve a cabo de una manera reflexiva, proyectiva y artesanal con el tiempo de dedicación necesario para evitar, en lo posible una reproducción irreflexiva, sistemática o mecánica y serial.

Estimar en si es conveniente que se contribuya en este sentido a aportar conocimiento sobre lo que supone a nivel individual la aceptación de un proyecto completamente natural, vital y complejo como es la de modelar la vida de una persona y que suponga, exponencialmente, el modelado de la cultura y la sociedad, o de alguna forma, dejar que los procesos discurran, "respetando" los procesos naturales, sin intervención ninguna.

La segunda alternativa es difícil de mantener cuando se interviene directamente en la vida de las personas desde tantos estamentos, lo que supone, en sí mismo una intervención directa sobre la reproducción humana y social.

\section{Referencias}

Alario Trigueros, María Teresa. 2009. Arte y feminismo. Donostia-San Sebastián: Nerea

Alonso, Mónica. 2015.”Arte, feminismo y maternidad “. Arte y Cultura Visual. http://www.m-arteyculturavisual.com/2015/03/19/arte-feminismo-y-maternidad/

Álvarez-Errecalde, Ana. 2005. "El nacimiento de mi hija”. Alvarezerrecalde.com, web personal de la artista. http://alvarezerrecalde.com/portfolio/el-nacimiento-de-mi-hija/?nacimiento?de?mi?hija/

Bosworth, Patricia. 1984. "Diane Arbus, her vision, lide, and death". The New York Times, 13 mayo. http://www.nytimes.com/1984/05/13/magazine/diane-arbus-her-vision-lide-anddeath.html?pagewanted=all

Butler, Judith. 1994. "Feminism by any other name [Rosi Braidotti]". Differences 6(2): 27-61 
Butler, Judith. 2002. “Los cuerpos que importan”. En: Cuerpos que importan: Sobre los limites materiales y discursivos del sexo, traducción, Alcira Bixio, 53-94. doi:10.4067/S071871812012000200027

Carrascosa Vacas, Alex. 2016. "La construcción del organismo social como una obra de arte (Repeating Beuys)". AusArt 4 (2): 23-34. doi:10.1387/ausart.17237

Castells Oliván, Manuel. 2002. La era de la información: Economía, sociedad y cultura. Vol. 1: La sociedad red. Versión castellana de Carmen Martínez Gimeno y Jesús Alborés. México DF: Siglo XII

Castells Oliván, Manuel. 2002. La era de la información: Economía, sociedad y cultura. Vol. 2: El poder de la identidad. Versión castellana de Carmen Martínez Gimeno y Jesús Alborés. México DF: Siglo

Cavendish, Lucy. 2013. "Elinor Carucci: The mother of all photographers". The Telegraph, 07 Oct www.telegraph.co.uk/women/mother-tongue/10350481/Elinor-Carucci-the-mother-of-all-photographers.html

Federici, Silvia. 2013. "Revolución en punto cero: Trabajo doméstico, reproducción y luchas feministas". Madrid: Traficantes de Sueños

Fernández Pujana, Irati. 2014. Feminismo y maternidad ¿una relación incómoda?: Conciencia y estrategias emocionales de mujeres feministas en sus experiencias de maternidad. Vitoria-Gasteiz: Emakunde. http://www.emakunde.euskadi.eus/contenidos/informacion/certamen_publicaciones/es_def/adjuntos/2013.feminismo.maternidad.relacion.incomoda.pdf

Fowler, William. 2015. "10,000 Revolutions: Meet Mary Kelly, the mother of all feminist artists". The Guardian, 18 mayo. https://www.theguardian.com/artanddesign/2015/may/18/mary-keIly-meet-the-mother-of-all-feminist-artists

García Palomo, Alberto. 2015. "El poder sexual de la maternidad: La activista María Llopis retrata el parto y la crianza de los bebés como un acto de insurgencia en el libro 'Maternidades subversivas'”. El País, 21 dic. http://elpais.com/elpais/2015/12/21/tentaciones/1450684816_941803.html?rel=cx_articulo\#cxrecs_s

Harrison, Margaret. 2014. "Tracey Emin is wrong on motherhood - having children doesn't mean you can't be a good artist too". The Independent, 5 oct. http://www.independent. co.uk/voices/comment/tracey-emin-is-wrong-being-a-mother-doesnt-mean-you-cant-be-agood-artist-too-9775997.html

Hudson, Mark. 2015. "The witch of St Ives: She is our greatest ever female artist but did Barbara Hepworth really 'bewitch ' fellow artists and discard husbands and children in relentless pursuit of perfection?". The Daily Mail, 13 junio. http://www.dailymail.co.uk/home/ event/article-3119835/Barbara-Hepworth-s-amazing-life-work-revealed.html

Iyengar, Udita. 2017. “Motherhood, raw and unfiltered”. The Psychologist, 9th June. https:// thepsychologist.bps.org.uk/moth erhood-raw-and-unfiltered

Legarretalza Iza, Matxalen. 2009. "El tiempo donado en el ámbito doméstico: Reflexiones para el análisis del trabajo doméstico y los cuidados". Cuadernos de Relaciones Laborales 26 (2): 45-69. doi:10.5209/CRLA.33400.

Monleón Pradas, Mau. 2010. "Hacia una visibilización de la crisis de los cuidados: Arte social frente a nueva esclavitud poscolonial”. Arte y Políticas de Identidad 2: 25-44. http://revistas.um.es/api/article/download/117261/110911 
Neuendorf, Henri. 2016. "Marina Abramović says children hold back female artists". Artnet, 25 julio. https://news.artnet.com/art-world/marina-abramovic-says-children-hold-back-female-artists- 575150

Pardo Sainz, Rebeca. 2012. "La maternidad en el arte: Visiones renovadas tras el Post Partum Document". En la retaguardia: Imagen, Identidad y memoria (blog), 6 junio. https://rebecapardo.wordpress.com/2012/05/06/la?maternidad?en?el?arte?visiones?renovadas?tras?el?post?partum?document/

Picard, Caroline. 2009. "I am searching for field character". The Green Lantern Press, A public notebook (blog), June 7, https://greenlanternpress.wordpress.com/2009/06/07/i-am-searching-for-field-character/

Puglise, Nicole. 2016. "Marina Abramović says having children would have been 'a disaster for my work'”. The Guardian, 26 julio. https://www.theguardian.com/artanddesign/2016/jul/26/ marina-abramovic-abortions-children-disaster-work

Rich, Adrienne. 1996. "Nacemos de mujer - la maternidad como experiencia e institución." Feminismos - Clásicos, 421

Rich, Adrienne. 1996. Nacemos de mujer : la maternidad como experiencia e institución. Postfacio a la edición española de Mercedes Bengoechea. Madrid : Cátedra

Tisdall, Caroline, ed. 1974. Art into Society, Society into Art: seven german artists (29 October 24 November 1974). London: ICA

Williams, Zoe. 2015. "Motherhood in art: From miracle milk to joke shop breasts and caesarean scars". The Guardian, 14 oct. https://www.theguardian.com/artanddesign/2015/oct/14/ motherhood-in-art-from-miracle-milk-to-joke-shop-breasts-and-caesarean-scars

Woodward, Richard B. 1992. "The disturbing photography of Sally Mann". The New York Times, 27 sept. http://www.nytimes.com/1992/09/27/magazine/the-disturbing-photography-of-sally-mann.html

\section{Webs visitadas}

Abramovic, Marina. 2016. Marinaabramovic.com, web personal de la artista. Última modificación, 11 julio. www.marinaabramovic.com

Arbus, Diane (1967) 2017. "Child Crying, New Jersey" Artne-Etherton Gallery. www.artnet.com/ artists/diane-arbus/child-crying-new-jersey-a-y_BEWmDbzh_fWqfEPpjG2A2

Carucci, Elinor. 2014. "Mother". Elinorcarucci.com, web personal de la artista. www.elinorcarucci.com/mother.php\#9

Casas Broda, Ana. 2013. "Kinderwunsch". Anacasasbroda.com, web personal de la artista. www.anacasasbroda.com/kinderwunsch

Emin, Tracey. 1998. "My bed". Traceyeminstudio.com, web personal de la artista. www.traceyeminstudio.com/artworks/1998/02/my-bed-1998-3/

Harrison, Margaret. 2017. Margaret-harrison.com, web personal de la artista. http://margaret-harrison.com/?gallery=work-2.

Hepworth, Barbara. (1927) 2017. "Mother and child."Barbarahepworth.org.uk, web personal de la fundación. http://barbarahepworth.org.uk/sculptures/1927/mother-and-child/ 
Hiller, Susan. 2017. Susanhiller.org, web personal de la artista. www.susanhiller.org/otherworks/ ten_months.html

Jones, Ann. 2012. "I wanted to love you more". ImageObjectText.com, web personal de la artista. https://imageobjecttext.com/2012/07/06/i?wanted?to?love?you?more/

Jones, Ann. 2012. "Motherhood as Art". ImageObjectText.com, web personal de la artista https://imageobjecttext.com/2012/05/06/motherhood?as?art/

Kelly, Mary. (1973-79) 2015. "Post partum document". Marykellyartist.com, web personal de la artista, última modificación 5 abril. www.marykellyartist.com/post_partum_document.html.

Kelly, Mary. (1975) 2004. "Post-Partum Document. Analysed markings and diary perspective schema (Experimentum Mentis III: Weaning from the Dyad)". The Tate Gallery 1984-86: Illustrated Catalogue of Acquisitions.... www.tate.org.uk/art/artworks/kelly-post-partum-document-analysed-markings-and-diary-perspective-schema-experimentum-t03925/text-catalogue-entry

Leibovitz, Annie. 2014. "Gwyneth Paltrow and Blythe Danne" Artnet Auctions. Sept 24th www. artnet.com/auctions/artists/annie-leibovitz/gwyneth-paltrow-and-blythe-danner-2

Llopis Navarro, Maria. 2010. "El embarazo como el mega estadio sexual de los cuerpos". MariaIlopis.com, web personal de la artista. www.mariallopis.com/portfolio/el-embarazo-como-el-mega-estadio-sexual-de-los-cuerpos/

Mann, Sally. 2017. "Family pictures". Sallymann.com web personal de la artista. http://sallymann.com/selected-works/family-pictures

Mann, Sally. 2017. Sallymann.com, web personal de la artista. http://sallymann.com/selected-works/southern-landscapes

Rispa, Aleydis. 2013. "Triple memòria”. Montoriol.com, web Galeria Esther Montoriol. http:// www.montoriol.com/aleydis-rispa-2/

Sabiá, Ana. (2013) 2017. "Madonnas contemporâneas 2012-13”. Anasabia.com, web personal de la artista. www.anasabia.com/madonnas-contemporaneas-2012-13

Sánchez, Bea. 2016. "No sé cómo puedes con todo II". Beasanchez.es, web personal de la artista. www.beasanchez.es/momisinthestudio/no-se-como-puedes-con-todo-ii/

Susan Sontag Foundation. 2010. Susansontag.com, web de la fundación. www.susansontag. $\mathrm{com} /$ SusanSontag/index.shtml

Viola, Bill. 1992. "Nantes triptych". The Tate Gallery 1984-86: Illustrated Catalogue of Acquisitions. www.tate.org.uk/art/artworks/viola-nantes-triptych-t06854

“¡Felices 50, Demi!: Desnuda y embarazada en Vanity Fair”. 2012. Vanity Fair Spain, 12 nov. www.revistavanityfair.es/celebrities/galerias/demi-moore-cumple-50-anos/8886/ image/593766

"Maris Bustamante". 2010. Reseña sobre la artista. Artecontraviolenciadegenero.org, 11 oct. http://artecontraviolenciadegenero.org/?p=2035

"Mónica Mayer." 2017. Pintomiraya.com, web personal de la artista. www.pintomiraya.com/pmr/ monica-mayer 


\section{Notas}

${ }^{1}$ Esta misma frase la utiliza Irate Fernández Pujana en el comienzo de Feminismo y maternidad ¿una relación incómoda?

2 "Con más consistencia se puede afirmar que la fuerza motriz de la economía mundial ha sido la capacidad del capitalismo internacional de apropiarse de las masas trabajadoras globales de campesinos expropiados y de amas de casa, es decir, de la inmensa cantidad de trabajo no contractual, incrementando asi de manera exponencial los porcentajes de extracción de plusvalía" (Federici 2013).

3 "I have three frames of reference ... my mother and father ... my own experience ... and the frame of reference of my children . the three are stuck together" (Louise Bourgeois, The complete prints, Motherhood \& Family. https://www.moma.org/explore/collection/lb/themes/ motherhood_family)

4 "Se llama Tecnología Social a la aplicación del conocimiento científico no a la materia inerte,sino a los sistemas sociales; este concepto es sinónimo de Tecnología Blanda, es decir, elconjunto de técnicas y métodos aplicados al desarrollo de las relaciones e interacciones humanas" (Carrascosa 2016, nota número 5).

5 "Un nuevo sistema de comunicación, que cada vez habla más un lenguaje digital universal, está integrando globalmente la producción y distribución de palabras, sonidos e imágenes de nuestra cultura y acomodándolas a los gustos de las identidades y temperamentos de los individuos. Las redes informáticas interactivas crecen de modo exponencial, creando nuevas formas y canales de comunicación, y dando forma a la vida a la vez que ésta les da forma a ellas" (Castells 2005).

6 "I am searching for field character", declaración de Beuys de 1973 (citado en Tisdal 1974, 48) (Carrascosa 2016, Nota número 4).

7 "Y propongo la hipótesis de que todas las tendencias de cambio que constituyen nuestro nuevo y confuso mundo están emparentadas y que podemos hallar sentido a su interrelación. Y, sí, creo, a pesar de una larga tradición de errores intelectuales a veces trágicos, que observar, analizar y teorizar es un modo de ayudar a construir un mundo diferente $y$ mejor" (Castells 2005).

8 "Fenómenos como el crecimiento de Internet, de los sistemas financieros, de la economía afectada por el envejecimiento y de la sociedad ecológica demuestran de forma evidente, que es necesario meditar sobre estas cuestiones y responder a ellas al mismo tiempo en sus dimensiones de inclusión e innovación. Los objetivos que Europa pretende alcanzar mediante este reto están clasificados en función del tipo de Sociedad al que van dirigidos: sociedades inclusivas; sociedades innovadoras y sociedades reflexivas" ("Europa en un mundo cambiante, sociedades inclusivas, innovadoras y reflexivas / Retos Sociales / Horizonte2020." 2017.. http://eshorizonte2020.es/retos-sociales/europa-en-un-mundo-cambiante-sociedades-inclusivas-innovadoras-y-reflexivas).

\footnotetext{
(Artículo recibido 29-04-17; aceptado 30-06-2017)
} 